\title{
One-Year Change in Locus of Control among People with Dementia
}

\author{
Ingeborg Halse ${ }^{a, b, c}$ Guro Hanevold Bjørkløf ${ }^{a}$ Knut Engedal ${ }^{a, b}$ \\ Geir Selbæk ${ }^{a, b, c}$ Maria Lage Barca ${ }^{a, b}$
}

aNorwegian National Advisory Unit on Ageing and Health, Vestfold Hospital Trust, Tønsberg, Norway; ${ }^{\text {b Department }}$

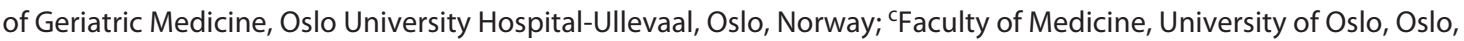
Norway

\section{Keywords \\ Dementia $\cdot$ Locus of control · Coping}

\begin{abstract}
Introduction: Knowledge of how perceptions of personal control change over time may provide valuable insights into how people cope with having dementia. The present study aimed to examine change in locus of control over a 12-month period in persons with dementia. Method: The study included 52 participants with dementia. Locus of control was measured with the Locus of Control of Behavior Scale (LoCB), with higher scores indicating a more external locus of control, interpreted as perceiving less personal control. $A \geq 5 \%$ change on the LoCB was considered clinically meaningful. We recorded sociodemographic characteristics and assessed dementia severity, cognition, ability to function independently in daily activities and physical self-maintenance, depressive symptomatology, and number of prescribed medications. Analyses were performed to examine differences between those with increases (more external) or decreases (less external) in the LoCB score after 12 months and to examine associations between baseline variables and change in the LoCB score. Results: The mean LoCB score for
\end{abstract}

the total sample did not change after 12 months (baseline mean 29.33 vs. follow-up mean 30.33, $p=0.553$ ); however, 2 subgroups emerged. Using the $\geq 5 \%$ cutoff revealed that the LoCB score changed for $92.3 \%$ of the sample, becoming less external (lower LoCB) for 21 participants and more external (higher LoCB) for 27 participants. At baseline, the mean LoBC score was higher in the group that became less external (33.81 vs. 24.56), $p=0.006$, while this was reverse at followup (23.57 vs. 34.41), $p=0.001$. Dementia severity and dependence in physical self-maintenance increased during the 12 months in both groups. Among those becoming more external, we also found a decline in cognition $(p=0.002)$, an increase in dependence in daily activities ( $p=0.003)$, an increase in the use of prescribed medication, and a decrease in depressive symptomatology ( $p=0.003)$. The baseline LoCB score was the only variable associated with 12-month change in LoCB scores ( $p=0.001)$. Conclusion: Most participants showed a clinically meaningful change in locus of control after 12 months. Those with more signs of dementia progression reported a decrease in personal control but also a decrease in depressive symptoms. These findings are interesting for our understanding of coping but must be replicated with a larger sample.

(c) 2021 The Author(s)

Published by S. Karger AG, Basel karger@karger.com www.karger.com/dee

Karger $\stackrel{\text { ' }}{=}$

BOPEN ACCESS
(C) 2021 The Author(s)

Published by S. Karger AG, Basel

This article is licensed under the Creative Commons Attribution 4.0 International License (CC BY) (http://www.karger.com/Services/ OpenAccessLicense). Usage, derivative works and distribution are permitted provided that proper credit is given to the author and the original publisher.
Correspondence to:

Ingeborg Halse, ingeborg.halse@ aldringoghelse.no 


\section{Introduction}

Coping is affected by how much a person feels in control of a situation. Having dementia means experiencing a decline in cognitive, instrumental, and social abilities, and these changes may require an almost ongoing coping response [1]. Information about how health practitioners can best assist in this coping process is important for enabling people with dementia to continue functioning in daily life. Knowledge about perceptions of control, regardless of actual control, may provide valuable information about how people with dementia cope with their challenges.

Perceived control is related to how we interpret a situation and our abilities to handle it and is thereby part of what motivates us to act [2-4]. It has been widely studied in health science, for example, among patients with depression, cancer, chronic illnesses, and pain, and is associated with treatment adherence, health behaviors, and adaptation to chronic diseases [5-8]. Studies have generally found that perceiving yourself to be in control of a situation is advantageous [9].

The concept of perceived control has also been studied among older people. Associations between coping and illness, cognitive decline, and mental health have been identified, with better health associated with greater perceptions of personal control [10]. The degree to which people perceived to be in control has been found to change in a curvilinear way with age, with a peak in midlife $[9,11,12]$. Decreases in perceptions of personal control with age could imply that older people may be more vulnerable when faced with adversities such as health problems [11].

Little research has been done on perceived control among people with dementia, however. A recent review reported on 18 studies investigating perceived control beliefs in this population, but still, only 6 studies examined change with a time span of at least 6 months [13]. Using the Pearlin Mastery scale [14], Burgener and Twigg [15] reported an increase in personal control over an 18-month period, while the remaining 5 studies did not report any statistically significant within-group changes [16-20].

Perceived control is an umbrella term that has been defined and operationalized in many ways [21], but one of the initial and most widely studied constructs is locus of control [22]. Locus of control is defined as the degree to which an individual expects what happens in life to be due to either internal or external causes. In other words, a person who expects things to happen in life because of himself or herself has an internal locus of control, and if the person expects things to happen in life because of powerful others or chance, fate, or luck, he or she has an external locus of control [22]. Thus, more external locus of control indicates less personal control. The aim of the present study was to examine if locus of control among people with dementia changes over a 1-year period, as well as which sociodemographic or clinical variables may be associated with such changes.

\section{Materials and Methods}

Design

The present prospective observational study is part of a larger study on the effectiveness and cost of the Norwegian day service program for people with dementia (ECOD). The ECOD study recruited 257 participants from 2013 to 2015 , and they were followed up for 2 years. The participants were interviewed annually either at home or at a daycare center. The study had substantial participant attrition from baseline to the 12-month follow-up, with 119 participants reaching either a study endpoint $(N=74$ moved to a long-term nursing facility, $N=13$ deceased), withdrawing $(N=$ $29)$, or were lost due to unknown causes $(N=3)$. For more details about the ECOD study, see the Rokstad et al. [23].

\section{Participants}

Participants with dementia were included if they were 65 years of age or older, had the revised Norwegian version of the Mini Mental Status Examination-NR (MMSE-NR) score of 15 or above, and resided at home. The dementia diagnosis was confirmed by 2 psychiatrists (coauthors K.E. and M.L.B.) using the ICD-10 criteria [24]. The current study reports on the participants who completed the Locus of Control of Behavior Scale (LoCB) at baseline and at the 12-month follow-up, or who responded to enough of the items to allow for statistical imputation. This resulted in LoCB data from 182 participants at baseline, 58 participants at follow-up, and a total of 52 participants with LoCB data at both timepoints (see flowchart, Figure 1).

Of the 52 participants included in this study, most were diagnosed with dementia due to Alzheimer's disease (71.2\%), followed by vascular dementia (13.5\%), mixed Alzheimer's/vascular dementia (5.8\%), Lewy body disease with dementia (3.8\%), Parkinson's disease with dementia (1.9\%), and other dementia diseases $(3.8 \%)$. The mean age was 80 years (SD 6.3), and $65.4 \%$ were women.

\section{Assessments}

Sociodemographic and clinical data such as age, sex, marital status, education, daycare attendance, use of prescription medication, and history of depression were recorded at baseline, and the participants were evaluated with the following instruments at both baseline and follow-up:

The LoCB is a self-reported questionnaire used to measure locus of control [25]. It consists of 17 Likert-style items, with a total score between 0 and 85 ; higher scores indicate a higher degree of external locus of control, interpreted as perceiving less personal control. The scale has been translated to Norwegian [26], and has been applied in several Norwegian studies with older participants $[27,28]$ and in studies with older people with depression [29] and dementia [30, 31]. 
Fig. 1. Flowchart of included participants and those lost to follow-up. LoCB, Locus of Control of Behavior Scale.

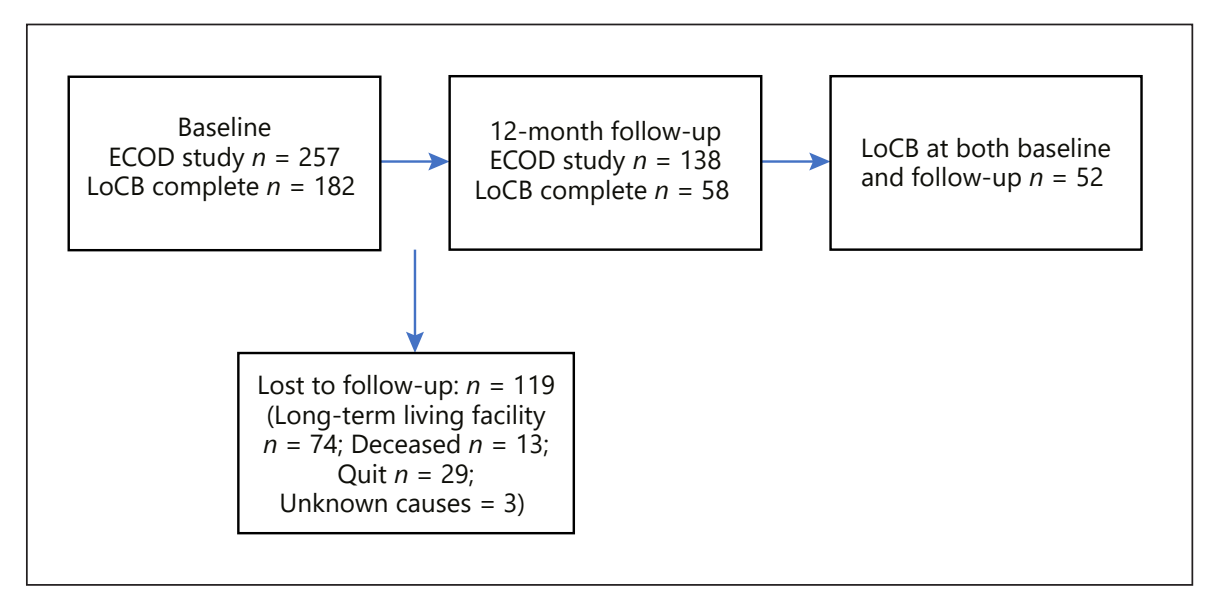

The MMSE-NR was used to measure global cognitive function. It consists of 20 items, and total scores vary between 0 and 30, with a higher score indicating better cognitive function $[32,33]$.

The Clinical Dementia Rating Scale (CDR) was used to assess dementia severity based on data collected from participants and contributing family carers. It comprises 6 items that assess severity of dementia, and the total score ranges from 0 to 18 (using the sum of boxes method), with 0 indicating no impairment and 18 indicating severe impairment $[34,35]$.

The Instrumental Activities of Daily Living Scale (IADL) and the Physical Self-Maintenance Scale (PSMS) were used to measure the ability to perform activities of daily life independently [36]. The IADL comprises 8 items, with a possible score between 8 and 31. The PSMS has 6 items, with a possible score between 6 and 30 . For both scales, a higher score indicates poorer independent functioning [36]. IADL and PSMS were proxy rated by a family caregiver.

The Montgomery-Aasberg Depression Rating Scale (MADRS) was used in an interview with the participant to assess the degree of depressive symptoms. It consists of 10 items, with possible scores ranging between 0 and 60 , with higher scores indicating more severe symptomatology [37]. The Norwegian version has been validated for use among people with dementia, and the best cutoff indicating depression was 7 points or higher [38].

\section{Statistics}

Statistical analyses were performed with the Statistical Package for the Social Sciences, version 27. The level of statistical significance was set at $p<0.05$. Missing values were imputed if cases had at least a $50 \%$ item response (relevant for LoCB and IADL). Missing values were imputed by random numbers drawn from an empirical distribution generated for each item of interest. Differences between those with baseline only $(N=130)$ versus baseline and follow-up measures of LoCB $(N=52)$ were assessed using $\chi^{2}$ test, independent samples $t$ test, or Mann-Whitney $\mathrm{U}$ test as appropriate.

We first examined the overall change in LoCB scores over 12 months among the 52 participants. Next, we dichotomized the participants into groups based on whether their LoCB score had increased or decreased $5 \%$ or more from baseline to follow-up. The developers of the LoCB suggested a $\geq 5 \%$ change as clinically sig- nificant because a change of this size toward internality was associated with both clinical improvement and reduced risk of relapse after treatment for a behavioral problem [39]. Based on dichotomization, we analyzed differences between those who became either more or less externally oriented at both baseline and followup, using independent samples $t$ test, $\chi^{2}$ test, or Mann-Whitney $U$ test as appropriate. Changes from baseline to follow-up within the groups were analyzed using paired samples' $t$ test or the Wilcoxon signed-rank test.

Finally, we conducted multiple linear regression analyses with 1 -year changes in LoCB scores (measure at 12 months - measure at baseline) as the dependent variable and baseline sociodemographic variables, number of prescribed medications, and scores on LoCB, CDR, MMSE-NR, IADL, PSMS, and MADRS as independent variables. First, unadjusted analyses were performed. Then, variables with a $p$ value of $<0.2$, together with the patients' age and sex, were included in the adjusted analysis. The model was assessed for multicollinearity, normality, and outliers.

\section{Results}

The 130 participants with only baseline LoCB response, compared to the 52 with a follow-up response, had at baseline more severe dementia (CDR score, median 6 [Q1:4.5-Q3:8] vs. 4.5 [Q1:4-Q3:7], $U=2,453.5$, $p=0.011$ ), had worse cognitive functioning (MMSE-NR score, median 20.5 [Q1:18-Q3:23] vs. 22.5 [Q1:20Q3:25], $U=4,568, p<0.001)$, and were more dependent in regard to both physical self-maintenance (PSMS score, median 9 [Q1:7-Q3:11] vs. 7 [Q1:6-Q3:9], $U=2,358.5$, $p=0.001)$ and instrumental activities of daily living (IADL score, median 22 [Q1:19-Q3:26] vs. 20 [Q1:16Q3:24.75], $U=2,452.5, p=0.008)$. Nonsignificant findings are not reported.

The mean LoCB score for the 52 participants did not change from baseline (mean 29.33, SD 11.97) to follow- 
Table 1. Patients' characteristics at baseline, and follow-up of those who became more or less externally oriented in 12 months

\begin{tabular}{|c|c|c|c|c|c|c|}
\hline \multirow[t]{2}{*}{ Characteristics } & \multicolumn{3}{|c|}{ Groups at baseline } & \multicolumn{3}{|c|}{ Groups at 12 months } \\
\hline & $\begin{array}{l}\text { less external } \\
(n=21)\end{array}$ & $\begin{array}{l}\text { more external } \\
(n=27)\end{array}$ & $p$ value & $\begin{array}{l}\text { less external } \\
(n=21)\end{array}$ & $\begin{array}{l}\text { more external } \\
(n=27)\end{array}$ & $p$ value \\
\hline Age, mean (SD) & $81.33(6.09)$ & $79.33(6.01)$ & $0.261^{1}$ & - & - & \\
\hline Female, $n(\%)$ & $16(76.2)$ & $16(59.3)$ & $0.355^{2}$ & - & - & \\
\hline Education ( $>10$ years), $n(\%)$ & $13(65.0)$ & $13(50.0)(N=26)$ & $0.473^{2}$ & - & - & \\
\hline Prior depression, $n(\%)$ & $6(28.6)$ & $5(18.5)$ & $0.634^{2}$ & - & - & \\
\hline Married, $n(\%)$ & $8(38.1)$ & $12(46.2)\left(N_{=26)}\right.$ & $0.796^{2}$ & $8(38.1)$ & $13(50)(N=26)$ & $0.602^{2}$ \\
\hline Attends day care, $n(\%)$ & $13(61.9)$ & $21(77.8)$ & $0.379^{2}$ & $\left.14(73.7){ }_{(} N=19\right)$ & $21(84.0)(N=25)$ & 0.643 \\
\hline Medications, $n$, mean (SD) & $4.52(3.06)$ & $5.22(2.58)$ & $0.395^{1}$ & $5.74(2.92)(N=19)$ & $6.13(2.44)(N=23)$ & $0.637^{1}$ \\
\hline CDR, mean (SD) & $5.14(2.57)$ & $5.21(2.31)$ & $0.924^{1}$ & $6.36(2.71)$ & $6.85(2.52)$ & $0.526^{1}$ \\
\hline MADRS, mean (SD) & $3.85(3.94)$ & $4.64(5.05)$ & $0.669^{3}$ & $3.43(4.70)$ & $3.30(4.61)$ & $0.915^{3}$ \\
\hline LoCB, mean (SD) & $33.81(12.18)$ & $24.56(10.27)$ & $0.006^{1}$ & $23.57(9.57)$ & $34.41(10.03)$ & $0.001^{3}$ \\
\hline MMSE-NR, mean (SD) & $21.95(2.96)$ & $23.22(3.13)$ & $0.160^{1}$ & $21.14(3.42)$ & $21.30(3.62)$ & $0.882^{1}$ \\
\hline PSMS, mean (SD) & $7.86(1.56)$ & $8.63(3.73)$ & $0.848^{3}$ & $8.76(2.30)$ & $9.28(3.65)(N=25)$ & $0.875^{3}$ \\
\hline IADL, mean (SD) & $19.48(5.48)$ & $19.59(5.77)$ & $0.944^{1}$ & $20.05(6.30)$ & $21.36(6.07)(N=25)$ & $0.476^{1}$ \\
\hline
\end{tabular}

CDR, Clinical Dementia Rating Scale; LoCB, the Locus of Control of Behavior scale; MMSE-NR, the revised Norwegian version of the Mini Mental Status Examination; IADL, the Instrumental Activities of Daily Living scale; PSMS, The Physical Self-Maintenance scale; MADRS, the Montgomery-Aasberg Depression Rating Scale. ${ }^{1}$ Independent sample $t$ test. ${ }^{2} \mathrm{X}^{2}$ test for independence. ${ }^{3}$ Mann-Whitney $U$ test.

Table 2. Changes from baseline to follow-up among those becoming more or less externally oriented in 12 months

\begin{tabular}{|c|c|c|c|c|c|c|}
\hline Variables & \multicolumn{3}{|c|}{ Less external $(N=21)$} & \multicolumn{3}{|c|}{ More external $(N=27)$} \\
\hline CDR, mean $(S D)$ Group that increased $(N=25)$ & $5.14(2.57)$ & $6.36(2.72)$ & $0.003^{1}$ & $5.02(2.14)$ & $6.80(2.56)$ & $0.001^{1}$ \\
\hline MADRS, mean $(S D)$ Group that decreased $(N=20) /$ Group that increased $(N=25)$ & $3.85(3.94)$ & $3.43(4.70)$ & $0.488^{2}$ & $4.64(5.05)$ & $3.30(4.61)$ & 0.0032 \\
\hline MMSE-NR, mean (SD) & $21.95(2.96)$ & $21.14(3.42)$ & $0.163^{1}$ & $23.22(3.13)$ & $21.30(3.62)$ & 0.0021 \\
\hline
\end{tabular}

CDR, Clinical Dementia Rating Scale; MMSE-NR, the revised Norwegian version of the Mini Mental Status Examination; IADL, the Instrumental Activities of Daily Living scale; PSMS, the Physical Self-Maintenance scale; MADRS, The Montgomery-Aasberg Depression Rating Scale. ${ }^{1}$ Paired samples' $t$ test. ${ }^{2}$ Wilcoxon signed rank test.

up (mean 30.33, SD 11.25), $p=0.553$. However, further examinations identified 2 subgroups with distinct changes. Using the criteria set by Craig et al. [25] to examine clinically meaningful changes in externality, the LoCB score remained stable for 4 participants, while 21 became less external (lower LoCB), and 27 became more external (higher LoCB). Participants in the group that became less external had a higher baseline LoCB score (mean 33.81, SD 12.18) than participants who became more external (mean 24.56, SD 10.27), $p=0.006$. At the follow-up, the participants who had become less external now had a lower LoCB score (mean 23.57, SD 9.57) than participants who had become more external (mean 34.41, SD 10.03), $p=0.001$. No other differences were found at baseline or at follow-up between the 2 groups (see Table 1). LoCB reliability analyses revealed a Cronbach's alpha value of 0.74 at baseline and 0.77 at follow-up.

From baseline to follow-up, both groups had an increase in dementia severity (CDR) and dependence in physical self-maintenance (PSMS). Those who became less external after 12 months had a CDR mean of 5.14 $(\mathrm{SD}=2.57)$ at baseline compared to $6.36(\mathrm{SD}=2.72)$ at follow-up, $p=0.003$, and a PSMS mean of 7.86 (SD = $1.56)$ at baseline compared to $8.76(\mathrm{SD}=2.30)$ at followup, $p=0.040$. Similarly, those who became more external after 12 months had a CDR mean of $5.02(\mathrm{SD}=2.14)$ at 
Table 3. Multiple linear regression analyses of associations between changes in LoCB and baseline patients' characteristics

\begin{tabular}{|c|c|c|c|c|c|}
\hline \multirow[t]{2}{*}{ Characteristics at baseline } & \multicolumn{2}{|l|}{ Unadjusted reg. analysis } & \multicolumn{3}{|l|}{ Adjusted reg. analysis } \\
\hline & $B(95 \% \mathrm{Cl})$ & $p$ value & $B(95 \% \mathrm{Cl})$ & $\beta$ & $p$ value \\
\hline Age & $-0.251(-0.805,0.103)$ & 0.127 & $-0.223(-0.649,0.202)$ & -1.138 & 0.425 \\
\hline Gender $($ female $=0 ;$ male $=1$ ) & $1.525(-4.561,7.612)$ & 0.617 & $2.185(-3.274,7.644)$ & 0.103 & 0.425 \\
\hline Civil status (unmarried $=0 ;$ married $=1$ ) & $1.963(-4.027,7.952)$ & 0.513 & & & \\
\hline Education $(<10$ years $=1 ;>10$ years $=2)$ & $-1.942(7.876,3.991)$ & 0.513 & & & \\
\hline Attends day care $($ no $=0 ;$ yes $=1)$ & $3.639(-2.675,9.953)$ & 0.252 & & & \\
\hline LoCB & $-0.428(-0.657,-0.199)$ & $<0.001$ & $-0.394(-0.628,-0.160)$ & -0.435 & 0.001 \\
\hline MADRS & $0.177(-0.519,0.872)$ & 0.611 & & & \\
\hline MMSE-NR & $0.694(-0.255,1.642)$ & 0.148 & $0.533(-0.325,1.391)$ & 0.158 & 0.217 \\
\hline CDR & $-0.316(-1.481,0.848)$ & 0.587 & & & \\
\hline IADL & $-0.117(-0.648,0.414)$ & 0.660 & & & \\
\hline PSMS & $0.241(-0.736,1.218)$ & 0.622 & & & \\
\hline Prescription medications, ${ }^{1} n$ & $0.345(-0.719,1.408)$ & 0.518 & & & \\
\hline Prior depression $($ no $=0 ;$ yes $=1)$ & $-2.773(-9.818,4.273)$ & 0.433 & & & \\
\hline$R^{2} 27.5 \%(F 4,46)=4.365, p=0.004$ & & & & & \\
\hline
\end{tabular}

CDR, Clinical Dementia Rating Scale; LoCB, the Locus of Control of Behavior scale; MMSE-NR, the revised Norwegian version of the Mini Mental Status Examination; IADL, the Instrumental Activities of Daily Living scale; PSMS, the Physical Self-Maintenance scale; MADRS, the Montgomery-Aasberg Depression Rating Scale. ${ }^{1}$ Potential ceiling effect, as maximum number reported was 9 different prescription medications.

baseline compared to $6.80(\mathrm{SD}=2.56)$ at follow-up, $p=$ 0.001 , and a PSMS mean of $8.63(\mathrm{SD}=3.73)$ at baseline compared to $9.28(\mathrm{SD}=3.65)$ at follow-up, $p=0.007$. Additional findings among those who became more external were an increase in the number of prescribed medications (baseline mean 5.00 [SD $=2.56]$ compared to follow-up mean 6.13 [SD $=2.44]$ ), $p=0.033$, greater dependence in instrumental activities of daily living (baseline mean 19.20 [SD $=5.80$ ] compared to follow-up mean 21.36 [SD $=6.07]$ ), $p=0.003$, a decline in cognitive function (baseline mean 23.22 [SD $=3.13$ ] compared to follow-up mean 21.30 [SD = 3.62]), $p=0.002$, and a decrease in depressive symptomatology (baseline mean $4.64[\mathrm{SD}=5.05]$ compared to follow-up mean 3.30 $[\mathrm{SD}=4.61]$ ), $p=0.003$ (see Table 2).

Finally, we investigated predictors of LoCB change (see Table 3). One participant with an extreme change in LoCB ( -45 points) was excluded from the regression analyses. LoCB at baseline $(p<0.001)$, age $(p=0.127)$, sex $(p=0.617)$, and MMSE-NR $(p=0.148)$ were included in the adjusted analysis. The adjusted model accounted for $27.5 \%$ of the variance of change in LoCB scores $(F 4,46)$ $=4.365, p=0.004$, and LoCB at baseline was the only independent variable associated with change in LoCB (standardized $\beta=-0.435, p=0.001$ ).

\section{Discussion}

To the best of our knowledge, this study is the first to examine change in locus of control among people with dementia. On average, LoCB sum scores did not change from baseline to follow-up; however, further examinations revealed substantial changes within the group. Using the criteria of $\geq 5 \%$ change as clinically meaningful showed that 48 participants (92.3\%) became either less $(n=21)$ or more $(n=27)$ externally oriented. Baseline LoCB was the only variable associated with a 12-month change in the LoCB sum score, and those becoming more externally oriented showed increases on more factors associated with dementia disease progression. Although the sample size was small, the findings suggest that locus of control among people with dementia varies across time and possibly in relation to dementia progression.

Adapting to the progression of a dementia disease aligns well with the theoretical notion that changes in locus of control are most likely to occur in novel situations [22]. Age-related findings suggest a general tendency toward feeling less personal control in old age $[9,11,12]$; however, approximately half of the current study sample showed the opposite. Our findings indicate that it is a faster progression of dementia, as indicated by worsening on several measures of dementia-related factors (CDR, 
MMSE-NR, IADL, and PSMS), that is related to experiencing loss of personal control, not age. Still, whether it is the speed of progression or the severity of the symptoms that is associated with loss of personal control remains unknown.

A consequence of dementia progression is to become more dependent on others to manage everyday activities. Thus, we suggest that those who reported less personal control after 12 months may have experienced more challenges as a result of their disease. However, shifting one's expectation of who is in control from oneself and instead place it externally, such as to family members and healthcare systems, may be a resourceful strategy for adaptive coping. The decrease in symptoms of depression observed in the group that became more externally oriented may support this, although a small sample size hinders early conclusions. Furthermore, we suggest that those who became less externally oriented managed to cope with living with the dementia disease, perhaps because of less challenges or experiences of mastery, and thereby gained an increased feeling of personal control. Though opposite, both ways agree with findings identified by Bjørkløf et al. [1], who reported in a review that reconstructing a sense of self and accepting support from family and friends were common coping strategies for people with dementia.

In Western societies, it is generally assumed that more personal control is associated with a healthier mental life, and studies have repeatedly found that having a more external locus of control is associated with adverse outcomes such as depression, anxiety, and decreased quality of life $[10,40]$. However, if objective control is indeed absent, then allowing oneself to not be responsible for what happens, such as with an external locus of control, can potentially be adaptive. Support for this is reported in studies examining chronic diseases such as cancer [41, 42]. Allowing oneself in such situations to trust others to make good decisions for oneself may be better than assuming all the responsibility alone.

There are limitations in this study, and the small number of participants is the most important. The present results must, therefore, be interpreted with caution. Because of this small number, we could not perform multiple regression analyses with the 2 groups separately. Finally, as the participants showed progression of dementia severity, one could potentially question the validity of the LoCB results at follow-up. However, as shown by Halse et al. [30], increases in dementia severity as indicated by the MMSE-NR affected the ability to complete the LoCB but not the reliability of the responses.
The major strengths of this study are the prospective design, the use of well-established assessment scales, and the fact that participants were diagnosed with dementia using standardized clinical criteria by 2 experts. Furthermore, the study examined clinically meaningful changes in the LoCB in addition to statistically significant changes. Statistical significance is vulnerable to both small and large sample sizes, and, arguably, can be less relevant for practitioners than reporting clinically meaningful results.

\section{Conclusion}

Most participants had a change in their locus of control, and those who became more external showed more signs of dementia disease progression. The findings may indicate that when the dementia progresses, shifting toward a greater trust in others or other external factors may allow for adaptive coping. However, the low number of participants prevents us from concluding based on the present data, and more research examining changes in locus of control in relation to dementia is needed.

\section{Acknowledgement}

The authors would like to acknowledge the daycare centers and in-home care units that participated in the study and those who participated in collecting the data. Finally, we are especially grateful to all the participants who volunteered to our study.

\section{Statement of Ethics}

The project has been accepted by the Regional Committee in Ethics in Medical Research in South-East Norway, REK SouthEast case No. 2013/1020. After being provided with written and oral information about the project, the participants were asked to give their written informed consent. Only participants with the capacity to give informed consent were included.

\section{Conflict of Interest Statement}

The authors have no conflicts of interest to declare.

\section{Funding Sources}

The data came from the ECOD project, which was funded by the Research Council of Norway (Grant No. 222083). 


\section{Author Contributions}

All authors jointly designed the study and participated in the statistical considerations and discussions of the results. I.H. performed the statistical analyses and wrote the manuscript, with continual input from the coauthors. All authors approved the final version of the manuscript.

\section{Data Availability Statement}

The data that support the findings of this study are available upon request from the corresponding author.

\section{References}

1 Bjørkløf GH, Helvik AS, Ibsen TL, Telenius EW, Grov EK, Eriksen S. Balancing the struggle to live with dementia: a systematic metasynthesis of coping. BMC Geriatr. 2019;19(1): 295.

2 Bandura A. Self-efficacy: toward a unifying theory of behavioral change. Adv Behav Res Ther. 1978;1(4):139-61.

3 Lazarus RS. Stress, appraisal, and coping. New York, NY: Springer Pub. Co; 1984.

4 Heckhausen J. Control behavior: psychological perspectives. In: Wrigth JD, editor. International encyclopedia of the social \& behavioral sciences. Elsevier Ltd; 2015.

5 Seeman TE, Unger JB, McAvay G, Mendes de Leon CF. Self-efficacy beliefs and perceived declines in functional ability: MacArthur studies of successful aging. J Gerontol B Psychol Sci Soc Sci. 1999 Jul;54(4):P214-22.

6 Wolinsky FD, Vander Weg MW, Martin R, Unverzagt FW, Willis SL, Marsiske M, et al. Does cognitive training improve internal locus of control among older adults? J Gerontol B Psychol Sci Soc Sci. 2009;65(5):591-8.

7 Janowski K, Kurpas D, Kusz J, Mroczek B, Jedynak T. Health-related behavior, profile of health locus of control and acceptance of illness in patients suffering from chronic somatic diseases. PLoS One. 2013;8(5):e63920.

8 Wallston KA. Control beliefs: health perspectives. In: Wrigth JD, editor. International encyclopedia of the social \& behavioral sciences. Elsevier Ltd; 2015. p. 819-21.

9 Robinson SA, Lachman ME. Perceived control and aging: a mini-review and directions for future research. Gerontology. 2017;63(5): $435-42$.

10 Bjørkløf GH, Engedal K, Selbæk G, Kouwenhoven SE, Helvik A-S. Coping and depression in old age: a literature review. Dement Geriatr Cogn Disord. 2013;35(3/4):121-54.

11 Infurna FJ, Gerstorf D, Zarit SH. Examining dynamic links between perceived control and health: longitudinal evidence for differential effects in midlife and old age. Dev Psychol. 2011;47(1):9-18.

12 Bonsaksen T, Lerdal A, Heir T, Ekeberg $\varnothing$, Skogstad L, Grimholt TK, et al. General selfefficacy in the Norwegian population: differences and similarities between sociodemographic groups. Scand J Public Health. 2019; 47(7):695-704.
13 Halse I, Bjørkløf GH, Engedal K, Selbæk G, Barca ML. Control beliefs among people with dementia: a systematic review. Dement Geriatr Cogn Disord. 2021;50(3):205-23.

14 Pearlin LI, Schooler C. The structure of coping. J Health Soc Behav. 1978;19(1):2-21.

15 Burgener S, Twigg P. Relationships among caregiver factors and quality of life in care recipients with irreversible dementia. Alzheimer Dis Assoc Disord. 2002;16(2):88-102.

16 Burgener SC, Buckwalter K, Perkhounkova Y, Liu MF. The effects of perceived stigma on quality of life outcomes in persons with earlystage dementia: longitudinal findings: Part 2. Dementia. Int J Soc Res Pract. 2015 Sep;14(5): 609-32.

17 Quinn C, Toms G, Jones C, Brand A, Edwards RT, Sanders F, et al. A pilot randomized controlled trial of a self-management group intervention for people with early-stage dementia (the SMART study). Int Psychogeriatr. 2016 May;28(5):787-800.

18 Hindle JV, Watermeyer TJ, Roberts J, Brand A, Hoare Z, Martyr A, et al. Goal-orientated cognitive rehabilitation for dementias associated with Parkinson's disease: a pilot randomised controlled trial. Int J Geriatr Psychiatry. 2018;33(5):718.

19 Stockwell-Smith G, Moyle W, Kellett U. The impact of early psychosocial intervention on self-efficacy of care recipient/carer dyads living with early-stage dementia: a mixed-methods study. J Adv Nurs. 2018;74(9):2167-80.

20 Clare L, Kudlicka A, Oyebode JR, Jones RW, Bayer A, Leroi I, et al. Individual goal-oriented cognitive rehabilitation to improve everyday functioning for people with early-stage dementia: a multicentre randomised controlled trial (the GREAT trial). Int J Geriatr Psychiatry. 2019;34:709-21.

21 Skinner EA. A guide to constructs of control. J Pers Soc Psychol. 1996;71(3):549-70.

22 Rotter JB. Generalized expectancies for internal versus external control of reinforcement. Psychol Monogr. 1966;80(1):1-28.

23 Rokstad AM, Halse I, Tretteteig S, Barca ML, Kirkevold $\varnothing$, McCabe L, et al. Effects and costs of a day care centre program designed for people with dementia: a 24 month controlled study. J Clin Trials. 2014;4:4.

24 WHO. The ICD-10 classification of mental and behavioural disorders: diagnostic criteria for research. Geneva: World Health Organization; 1993.
25 Craig AR, Franklin JA, Andrews G. A scale to measure locus of control of behaviour. $\mathrm{Br} \mathrm{J}$ Med Psychol. 1984;57(Pt 2)(2):173-80.

26 Nordtug B, Krokstad S, Holen A. Personality features, caring burden and mental health of cohabitants of partners with chronic obstructive pulmonary disease or dementia. Aging Ment Health. 2011 Apr;15(3):318-26.

27 Bruvik FK, Ulstein ID, Ranhoff AH, Engedal $\mathrm{K}$. The effect of coping on the burden in family carers of persons with dementia. Aging Ment Health. 2013;17(8):973-8.

28 Helvik AS, Bjørkløf GH, Corazzini K, Selbæk G, Laks J, Østbye T, et al. Are coping strategies and locus of control orientation associated with health-related quality of life in older adults with and without depression? Arch Gerontol Geriatr. 2016;64:130-7.

29 Bjørkløf GH, Engedal K, Selbæk G, Maia DB, Coutinho ESF, Helvik AS. Locus of control and coping strategies in older persons with and without depression. Aging Ment Health. 2016;20(8):831-9.

30 Halse I, Bjørkløf GH, Engedal K, Rokstad AMM, Persson K, Eldholm RS, et al. Applicability of the locus of control of behaviour scale for people with dementia. Aging Ment Health. 2020;24(12):2111-6.

31 Halse I, Bjørkløf GH, Engedal K, Selbæk G, Barca ML. Locus of control and its associations with depressive symptoms amongst people with dementia. Dement Geriatr Cogn Disord. 2021;50(3):258-65.

32 Folstein MF, Folstein SE, McHugh PR. "Minimental state区. A practical method for grading the cognitive state of patients for the clinician. J Psychiatr Res. 1975;12(3):189-98.

33 Strobel C, Engedal K. MMSE-NR. Norsk Revidert Mini Mental Status Evaluering. Revidert og utvidet manual. Nasjonal Kompetansetjeneste for Aldring og Helse; 2008.

34 Hughes CP, Berg L, Danziger WL, Coben LA, Martin RL. A new clinical scale for the staging of dementia. Br J Psychiatry. 1982;140(6): 566-72.

35 O'Bryant SE, Waring SC, Cullum CM, Hall J, Lacritz L, Massman PJ, et al. Staging dementia using clinical dementia rating scale sum of boxes scores: a Texas Alzheimer's research consortium study. Arch Neurol. 2008;65(8): $1091-5$. 
36 Lawton MP, Brody EM. Assessment of older people: self-maintaining and instrumental activities of daily living. Gerontologist. 1969; 9(3):179-86.

37 Montgomery SA, Asberg M. A new depression scale designed to be sensitive to change. Br J Psychiatry. 1979;134(4):382-9.

38 Knapskog AB, Barca ML, Engedal K. A comparison of the validity of the Cornell Scale and the MADRS in detecting depression among memory clinic patients. Dement Geriatr Cogn Disord. 2011;32(4):287-94.
39 Craig A, Andrews G. The prediction and prevention of relapse in stuttering: the value of self-control techniques and locus of control measures. Behav Modif. 1985;9(4):427-42.

40 Benassi VA, Sweeney PD, Dufour CL. Is there a relation between locus of control orientation and depression? J Abnorm Psychol. 1988; 97(3):357-67.
41 Burish TG, Carey MP, Wallston KA, Stein MJ, Jamison RN, Lyles JN. Health locus of control and chronic disease: an external orientation may be advantageous. J Soc Clin Psychol. 1984;2(4):326-32.

42 Brown AJ, Thaker PH, Sun CC, Urbauer DL, Bruera E, Bodurka DC, et al. Nothing left to chance? The impact of locus of control on physical and mental quality of life in terminal cancer patients. Support Care Cancer. 2017 Jun;25(6):1985-91. 